\title{
Gastric Adenoma with Low-Grade Dysplasia: Two Countries, Two Outcomes
}

\author{
Sun-Young Lee
}

Received: 22 August 2013/Accepted: 23 August 2013/Published online: 20 September 2013

(C) Springer Science+Business Media New York 2013

The use of endoscopic submucosal dissection (ESD) for the treatment of gastric neoplasms is rapidly increasing as judged by the large number of publications on the topic (1925 publications $1 / 1 / 2000-8 / 8 / 2013) .{ }^{1}$ Of these 1,925 publications, more than half originate from the countries with a high prevalence of gastric neoplasia such as South Korea and Japan (Fig. 1). To interpret this literature, the reader should know that the indications for ESD differ between two countries: gastric adenomas with either lowgrade dysplasia (LGD) or high-grade dysplasia (HGD) are usually endoscopically resected in Korea, whereas ESD is mostly reserved for early gastric cancers (EGCs) in Japan. This widely different treatment strategy is not related to fundamental differences in the intent of endoscopic therapy or major technical differences between the two countries, but rather due to marked differences in histologic classification, since gastric adenoma with dysplasia diagnosed by a Korean pathologist would be likely diagnosed as an EGC in Japan.

In a Korean study performed by Kim et al. [1] reported in this issue of Digestive Diseases and Sciences, complete endoscopic resection revealed that 22 of 285 gastric adenomas initially reported as LGD by endoscopic biopsy were reclassified as having HGD, with 24 lesions reported as adenocarcinomas. They reported that a lesion size $\geq 2 \mathrm{~cm}$, the presence of erythema, or depressed lesions were significantly associated with an upgraded histology, consistent with previous Korean studies reporting that EGC diagnosed initially with endoscopic biopsy can be

S.-Y. Lee $(\square)$

Department of Internal Medicine, Konkuk University School of Medicine, 120-1 Neungdong-ro, Gwangjin-gu, Seoul 143-729, South Korea

e-mail: sunyoung@kuh.ac.kr underdiagnosed as LGD [2-5]. Yet, in Japan, where the term "adenoma with low-grade atypia" is used to describe a lesion, concerns might be raised about the endoscopic resection of adenomas with LGD.

Although a high incidence of gastric neoplasm exists in Korea and Japan due to the high prevalence of Helicobacter pylori infection, the diagnosis, treatment, and prognosis differ between countries (Fig. 2). Japanese pathologists are reluctant to use the term "gastric adenoma with LGD" because in Japan "dysplasia" connotes "cancer". The term "adenoma with low-grade atypia" has been substituted for "dysplasia" in Japan because the Japanese histological classification of gastric well-differentiated tumors divides intraepithelial gastric neoplasia into adenoma or carcinoma with low and high-grade cytological atypia, unlike the Western criteria [6-9]. From the Japanese viewpoint, gastric adenomas with LGD diagnosed by Western criteria include typical adenomas of the small intestinal type and gastric foveolar type which often have papillary and tubular structures, and thus diagnosed as "carcinoma without invasion" in Japan. Therefore, lesions diagnosed as gastric adenomas in Japan rarely progress to cancer [10], unlike adenomas diagnosed in other countries $[11,12]$. In Korea, pathological diagnosis is based on the Western criteria, and thus gastric neoplasia is classified as LGD, HGD, or adenocarcinoma, as reported by Kim et al. [1]. As a consequence, lesions with the same diagnosis are treated differently in Korea and in Japan due to differing histology.

The second concern relates to category 4 Vienna classifications for gastrointestinal epithelial neoplasia: 4.1 is HGD, 4.2 is noninvasive carcinoma in situ, and 4.3 is suspicious for invasive carcinoma with intramucosal

\footnotetext{
${ }^{1}$ Institute for Scientific Information Web of Science.
} 


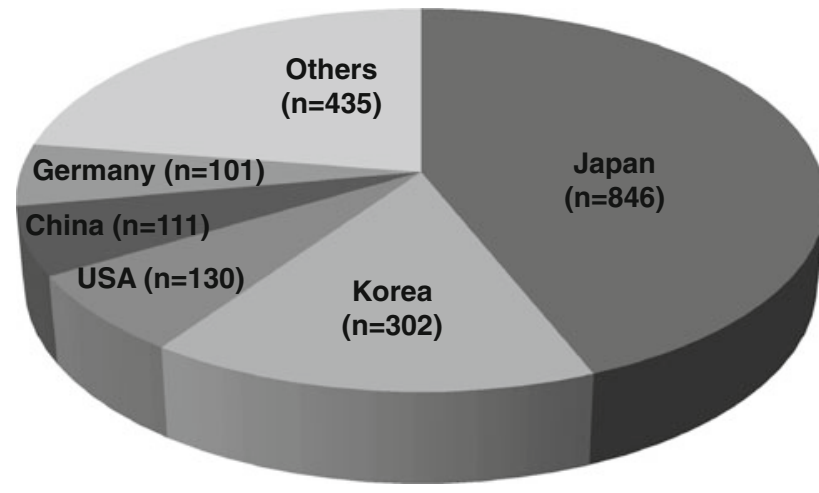

Fig. 1 Publications on endoscopic submucosal dissection according to the countries. Since 2000 , there were 1,925 publications in SCI expanding SSCI, and A\&HCI journals. Most of the papers were published from countries where there is a high incidence of gastric neoplasm due to the high prevalence of Helicobacter pylori infection

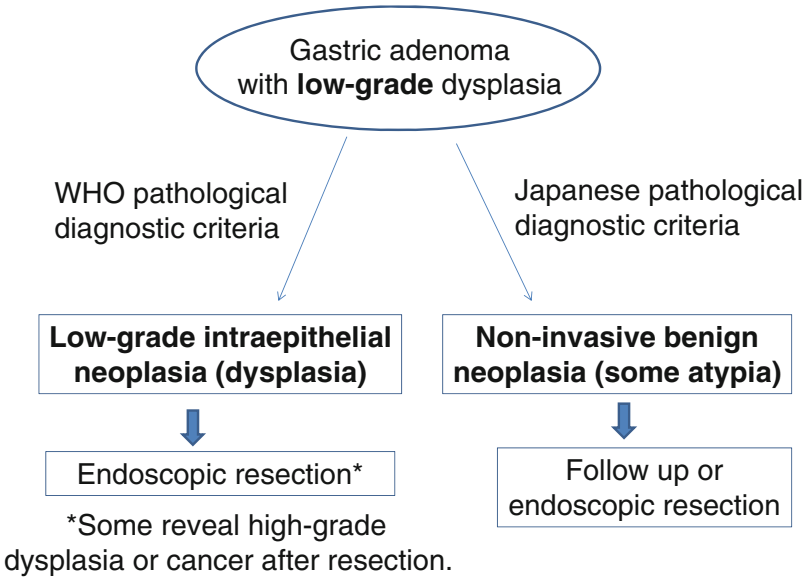

Fig. 2 Different diagnosis and management for gastric adenoma with low-grade dysplasia. According to the Japanese classification of gastric carcinoma, low-grade dysplasia is closer to group 3 (benign neoplasia) and some of group 2 (atypical epithelium which cannot be diagnosed as neoplastic atypia) lesions. Therefore, follow up is preferred to endoscopic resection in Japan

carcinoma [8]. Dysplasia is a precancerous lesion associated with a carcinoma risk increasing in parallel with the histological grade [9]. Most Western pathologists use the term "dysplasia" to describe a neoplastic premalignant abnormality; evidence of invasion into the lamina propria is needed to diagnose cancer. Nonetheless, while most Western pathologists require a structurally invasive focus for a cancer diagnosis, in the Japanese classification, there is no formal adenocarcinoma in situ classification [8]. The fundamental concepts for the diagnosis of cancer are completely different between WHO classification and Japanese classification. Japanese pathologists diagnose cancer based on severe cytologic dysplasia, irrespective of the presence of invasion $[6,13]$. Therefore, a considerable number of non-invasive intramucosal (intraepithelial)

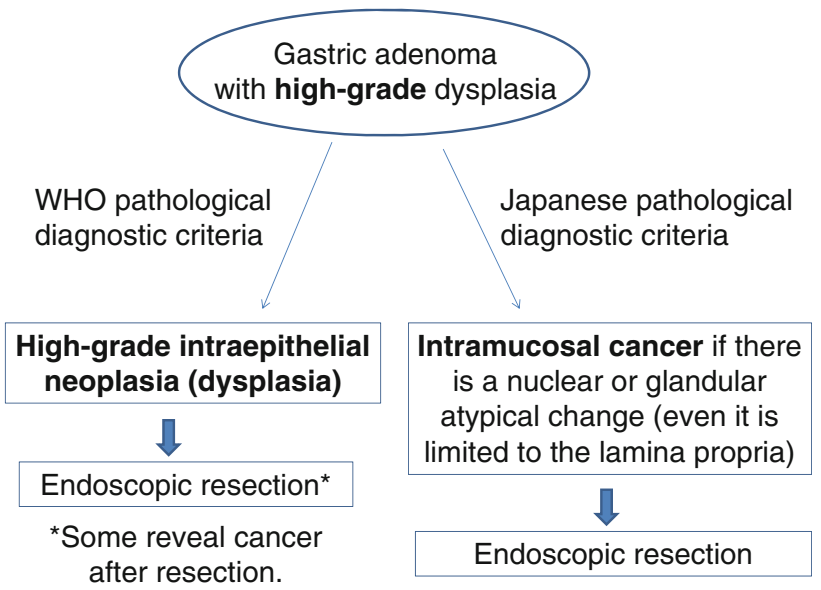

Fig. 3 Different diagnosis and management for gastric adenoma with high-grade dysplasia. Japanese pathologists are reluctant to use the term "high-grade dysplasia" for the gastric lesions, and therefore, it is often diagnosed as intramucosal carcinoma in Japan

gastric neoplasms, so-called HGD outside Japan, are resected by ESD after being diagnosed as EGC in Japan. Although the Vienna classification was designed to reduce diagnostic discrepancies with Japan, it has been difficult to adopt due to differing criteria used to diagnose gastric epithelial neoplastic lesions $[6,8]$. Thus, the prevalence of EGC is inflated in Japan since lesions that most Western pathologists identify as HGD are often considered intramucosal adenocarcinomas in Japan (Fig. 3).

In addition to the inconsistency between the countries regarding the diagnosis of EGC, the grading system for EGC is not the same in Japan as elsewhere [6-9, 13]. There are discrepancies with respect to the depth of invasion and the EGC cell types. For example, when cancer cells are limited to the first submucosal (SM1) layer with microvascular invasion (i.e., lymphatic, venous, or perineural invasion) in the third submucosal (SM3) layer, the depth of invasion is identified as the "SM1 layer with microvascular invasion" outside Japan but as the "SM3 layer" within Japan. Based on the concept that one cannot completely distinguish between microvascular invasion and true direct invasion (even if there is a distance between the tumor mass and distinct lymphatic/ vascular invasion), the depth of cancer invasion is defined as the deepest level of the gastric wall in Japan. Nonetheless, when an EGC is $80 \%$ moderately-differentiated adenocarcinoma and $20 \%$ poorly-differentiated adenocarcinoma, the main diagnosis outside Japan would be the dominant cell type of "moderately-differentiated adenocarcinoma", whereas some Japanese pathologists define the diagnosis "poorly-differentiated adenocarcinoma" based on the most advanced cell type. While neither diagnostic criteria can be considered right or wrong, Japanese diagnoses for gastric neoplasms are usually of higher grade than for comparable lesions diagnosed in other countries. 
The final concern is the need for a unified therapeutic plan for gastric dysplasia. In countries such as Japan where the term "low and high-grade atypia" are used instead of "LGD and HGD" for a gastric adenoma, ESD is mostly indicated for EGC, including intramucosal carcinomas, which would be considered severe dysplasia or HGD outside of Japan. On the contrary, in countries where gastric adenomas are classified as either LGD or HGD, as in the study by Kim et al. [1], ESD is indicated not only for gastric adenomas with HGD but also for those with LGD.

In conclusion, the diagnosis of gastric adenoma with LGD in Korea carries a substantial risk of cancer development, as reported by Kim et al. [1]. Efforts should be made to develop unified international guidelines for the management of gastric adenoma with LGD by overcoming the differences in the diagnostic criteria that are currently used in different countries and lead to a different natural course.

Acknowledgments This work was supported by the National Research Foundation of Korea funded by the Korean Government (NRF 2012K2A2A4010622). The author thanks to Dr. Yasuo Ohkura at the Department of Pathology, Kyorin University School of Medicine, Tokyo, Japan and Dr. Tetsuo Nemoto at the Department of Surgical Pathology, School of Medicine, Toho University, Tokyo, Japan for their valuable comments in revising Fig. 1 and descriptions on the Japanese criteria.

\section{Conflict of interest None.}

\section{References}

1. Kim MK, Jang JY, Kim JW, et al. Is lesion size an independent indication for endoscopic resection of biopsy-proven low-grade gastric dysplasia? Dig Dis Sci. (Epub ahead of print). doi: 10 . 1007/s10620-013-2805-8.

2. Choi CW, Kang DH, Kim HW, et al. Endoscopic submucosal dissection as a treatment for gastric adenomatous polyps: predictive factors for early gastric cancer. Scand J Gastroenterol. 2012;47:1218-1225.

3. Cho SJ, Choi IJ, Kim CG, et al. Risk of high-grade dysplasia or carcinoma in gastric biopsy-proven low-grade dysplasia: an analysis using the Vienna classification. Endoscopy. 2011;43: 465-471.

4. Kim YJ, Park JC, Kim JH, et al. Histologic diagnosis based on forceps biopsy is not adequate for determining endoscopic treatment of gastric adenomatous lesions. Endoscopy. 2010;42: 620-626.

5. Sung HY, Cheung DY, Cho SH, et al. Polyps in the gastrointestinal tract: discrepancy between endoscopic forceps biopsies and resected specimens. Eur J Gastroenterol Hepatol. 2009;21: 190-195.

6. Schlemper RJ, Riddell RH, Kato Y, et al. The Vienna classification of gastrointestinal epithelial neoplasia. Gut. 2000;47: 251-255.

7. Tamura W, Fukami N. Early gastric cancer and dysplasia. Gastrointest Endosc Clin N Am. 2013;23:77-94.

8. Stolte M. The new Vienna classification of epithelial neoplasia of the gastrointestinal tract: advantages and disadvantages. Virchows Arch. 2003;442:99-106.

9. Lauwers GY, Srivastava A. Gastric preneoplastic lesions and epithelial dysplasia. Gastroenterol Clin North Am. 2007;36: 813-829.

10. Yamada H, Ikegami M, Shimoda T, et al. Long-term follow-up study of gastric adenoma/dysplasia. Endoscopy. 2004;36:390-396.

11. Rugge M, Cassaro M, Di Mario F, et al. The long term outcome of gastric noninvasive neoplasia. Gut. 2003;52:1111-1116.

12. Park SY, Jeon SW, Jung MK, et al. Long-term follow-up study of gastric intraepithelial neoplasias: progression from low-grade dysplasia to invasive carcinoma. Eur J Gastroenterol Hepatol. 2008;20:966-970.

13. Japanese Gastric Cancer Association. Japanese classification of gastric carcinoma: 3rd English edition. Gastric Cancer. 2011;14: $101-112$. 